\title{
Vocal Cord Paralysis -An Etiological Analysis
}

\author{
Architha Menon ${ }^{1 *}$, Rehna Mohamed ${ }^{2}$, Vidhya Chemmanchery ${ }^{2}$, Soumya Vedavyasa ${ }^{2}$ and Borlingegowda Viswana- \\ tha $^{3}$
}

${ }^{1}$ Senior Resident, Department of ENT, Bangalore Medical College \& Research Institute, India

${ }^{2}$ Postgraduate, Department of ENT, Bangalore Medical College \& Research Institute, India

${ }^{3}$ Professor and Head, Department of ENT, Bangalore Medical College \& Research Institute, India

*Corresponding author: Architha Menon P, Senior Resident, Department of ENT, Bangalore Medical College\& Research Institute, Bangalore, India

\begin{abstract}
Introduction: Vocal cord paralysis is the total interruption of nerve impulse resulting in absence of laryngeal muscle movement. The most common type of vocal cord paralysis found is due to recurrent laryngeal nerve injury. The pathology can be either along the course of the tenth cranial nerve from cerebral cortex to neuromuscular junction or could be from mechanical fixation of cricoarytenoid joint.
\end{abstract}

Objective: To study the various etiological factors contributing to vocal cord paralysis.

Materials and methods: Patients with vocal cord paralysis [unilateral and bilateral] who were treated at a tertiary institute for a period of 3 years were included. The immobility of cords was assessed with the use of indirect laryngoscopy via mirror or rigid angled telescopy or flexible laryngoscopy. The patients were categorized based on laterality of paralysis as well as based on various etiological factors into groups. Appropriate statistical analysis was done.

Results: Among 50 patients, $84 \%$ cases had unilateral vocal cord paralysis. Surgical cause was found to be the most common etiology of unilateral vocal cord paralysis (34\%) closely followed by idiopathic (18\%) and neoplasm (16\%). In bilateral vocal cord paralysis, surgical and neurological causes were found to be the main causative factor.

Conclusion: Vocal cord paralysis has a wide range of factors contributing to its occurrence with surgical cause being a common one. A comprehensive assessment of patient should be performed to determine the cause which facilitates a direct treatment plan.

Keywords: Vocal cord paralysis; etiology

Abbreviations: VCP: Vocal Cord Paralysis; RLN: Recurrent Laryngeal Nerve; UVCP: Unilateral Vocal Cord Paralysis

\section{Introduction}

Vocal cord paralysis [VCP] is the total interruption of nerve impulse resulting in absence of laryngeal muscle movement. Vocal cord paresis implies the hypo function or hypo mobility secondary to neurologic injury, whereas vocal cord paralysis is the complete immobility of a muscle. The most common type of vocal cord paralysis found is due to recurrent laryngeal nerve injury [RLN].The left recurrent laryngeal nerve branch is more frequently involved as the intrathoracic course of left RLN is twice more than the right RLN [1]. Left RLN travels into mediastinum and loops around arch of aorta prior to penetrating larynx more vertically. Right RLN loops around subclavian artery and has a shorter course \& returns and penetrates horizontally. Paralysis of the left vocal cord is reported 1.4-2.5 times more than right [2]. There are multiple ways of classification of vocal cord paralysis, namely:
a) Unilateral or bilateral
b) Abductor or adductor or both
c) Complete or incomplete 
d) Congenital or acquired

e) Sensory or motor or combined

The clinical presentation is based on the type of classification. The most common symptom is voice change, the severity and degree of which will depend on the position of the vocal cords [34]. Other symptoms are stridor, which is seen in bilateral abductor paralysis, aspiration which is usually noted in bilateral adductor paralysis, dysphagia, sore throat, cough. The voice quality also varies as per the type of paralysis. Unilateral vocal cord paralysis can be asymptomatic also.

a. Forced whisper- organic adductor paralysis.

b. Faint whisper - functional adductor paralysis.

c. Normal voice morning \& tires as the day goes by unilateral abductor palsy.

Diagnosis is made mainly through indirect laryngoscopic examination. Early detection is remarkable as late diagnosis could result in serious symptoms or even death $[2,5]$. The pathology can be either along the course of the tenth cranial nerve from cerebral cortex to neuromuscular junction or could be from mechanical fixation of cricoarytenoid joint. This study aims to assess the contemporary causative factors for vocal cord paralysis other than laryngeal or hypopharyngeal malignancies in a tertiary setup in the state of Karnataka in India.

\section{Materials and Methods}

Patients with vocal cord paralysis [unilateral and bilateral] from a tertiary institute for a period of 3 years were included in the study. The immobility of cords was assessed with the use of indirect laryngoscopy via mirror or rigid angled telescopy or flexible laryngoscopy. The patients were categorized based on laterality of paralysis as well as based on various etiological factors into groups. Children less than 15 years of age and inadequate documentation of VC immobility were excluded from the study.

\section{Results}

A total number of 50 cases were included in the study. Among which 22 were males and 28 were females. The range of age group was between 21 to 72 years with a mean age of 56 years. $84 \%$ were unilateral vocal cord paralysis [UVCP] and $16 \%$ were bilateral vocal cord paralysis [BVCP] (Figure 1). Among the surgical causes, maximum incidence was seen after thyroidectomy [n=12] $64 \%$ followed by oesophageal surgeries and cardiovascular thoracic surgeries like carotid endarterectomy or mediastinal surgeries etc. Bronchogenic cancer predominated the malignancy category $[\mathrm{n}=5$ ] followed by oesophageal malignancy [ $\mathrm{n}=3$ ]. In bilateral vocal cord paralysis, $62 \%[\mathrm{n}=5]$ was attributed to surgical trauma [Total thyroidectomy]

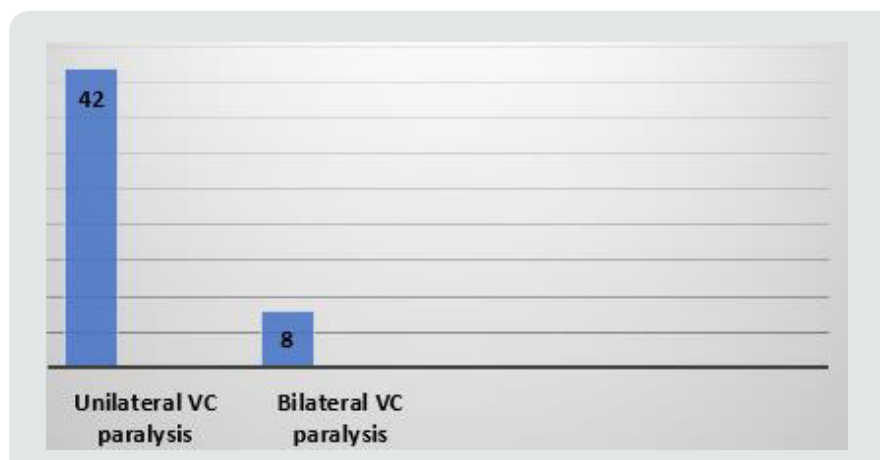

Figure 1: Laterality of Vocal Cord Paralysis.

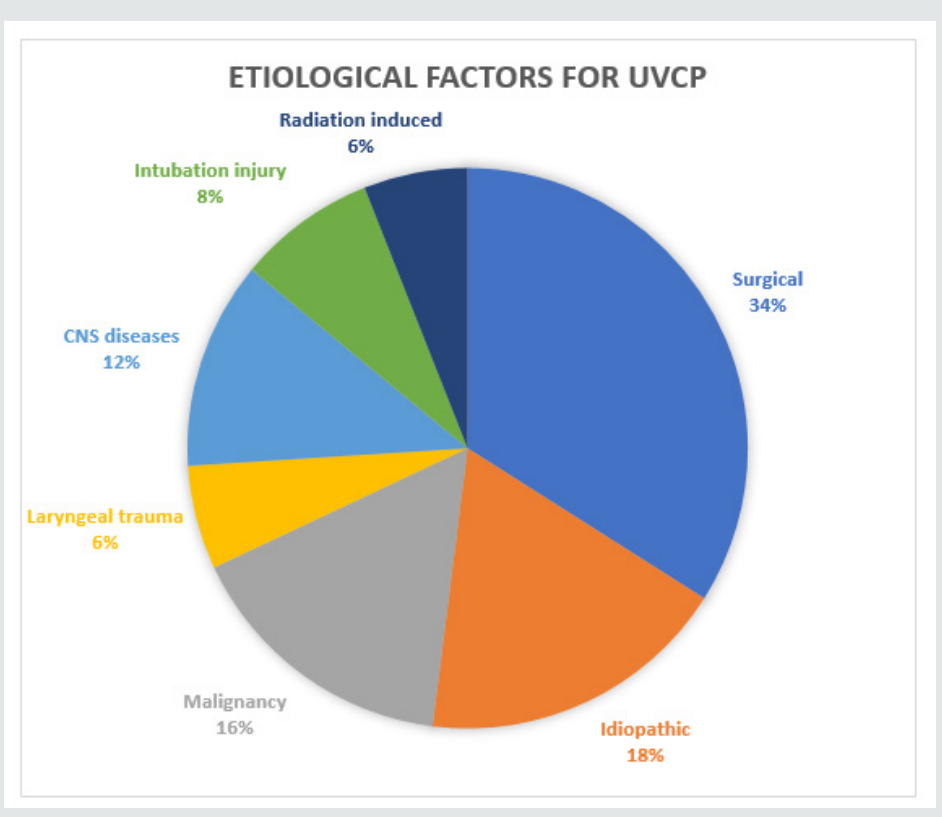

Figure 2. 


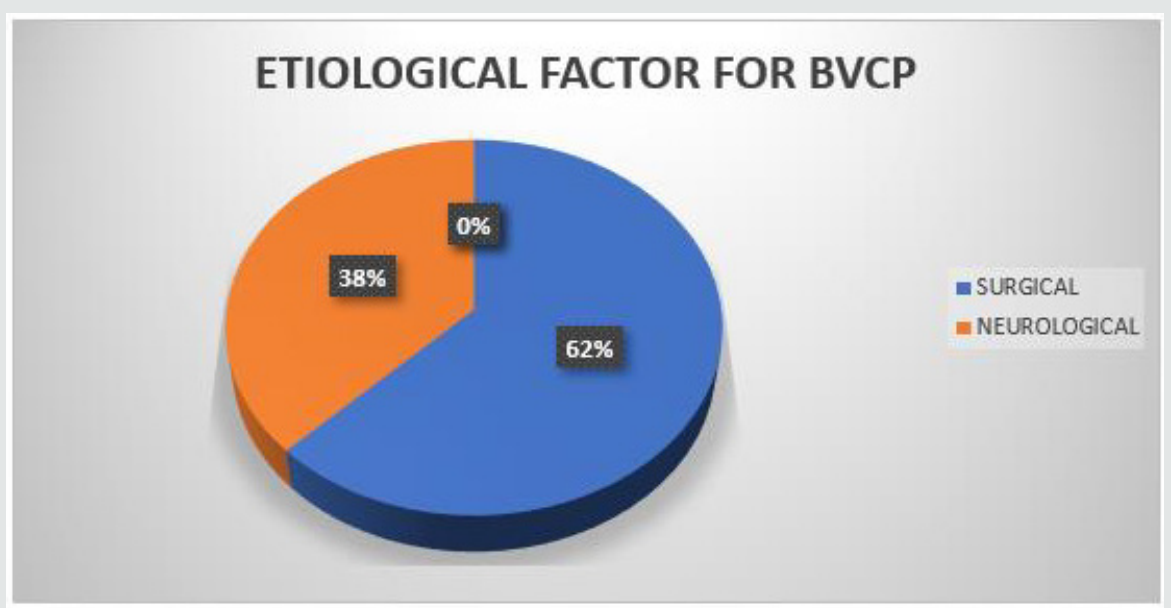

Figure 3.

\section{Discussion}

Vocal cord paralysis is a common feature of some underlying disease entity which can even be life threatening at times. Hence identifying the etiology of vocal cord paralysis plays a significant role in prognosis and treatment outcomes. The clinical presentation depends on the site and severity of injury $[6,7]$. The age group of 51-60 years is found to be more prone for vocal cord paralysis, probably due to history of chronic smoking and the higher occurrence of malignancy and systemic co morbidities like diabetes and hypertension as per Gupta et al. [8,9]. Our study was also in accordance with the above study wherein average age was found to be 56 yrs. A comparative analysis of incidence of unilateral and bilateral vocal cord paralysis is shown in the table below [Table
1]. Surgery, neoplasm, and idiopathic causes have been quoted in literature as the common culprits for the occurrence of vocal cord paralysis [Table 2]. Neoplasm was found to be the most common etiology in earlier studies $[3,10,11]$. Our study also reinforces the same with surgical cause being the predominant one. The bright side of post thyroidectomy cases of UVCP is the high rate of complete recovery, almost $93 \%$ as per studies [12]. The presence of adhesions, anatomical derangement in recurrent goitres and vascular masses obscuring surgical field due to excessive bleeding are few predisposing factors rendering the identification of the recurrent laryngeal nerve cumbersome intra-operatively $[9,12]$. Other surgeries like oesophageal surgery, cervical spine surgeries, and cardiac surgeries still pose a high risk of VC palsy $[1,12,13]$.

Table 1: Comparison of laterality.

\begin{tabular}{|c|c|c|c|}
\hline Study & Sample Size & UVCP (\%) & BVCP (\%) \\
\hline Chen [10] & 291 & 89 & 11 \\
\hline Laura [1]] & 435 & 83 & 17.56 \\
\hline Feehery [7] & 482 & 84.43 & 4.48 \\
\hline Titche [3] & 134 & 95.52 & 8.64 \\
\hline Leder [12] & 81 & 91.35 & 14 \\
\hline Parnell [13] & 100 & 86 & 16 \\
\hline Present study & 50 & 84 & \\
\hline
\end{tabular}

Table 2: Etiology of unilateral vocal cord paralysis- comparative analysis.

\begin{tabular}{|c|c|c|c|c|c|}
\hline Author & Number of Cases(n) & Surgery \% & Idiopathic \% & Neoplasm \% & Trauma \% \\
\hline Yumoto et al. [13] & 422 & 33 & 22 & 19 & 32 \\
\hline Ramadan et al. [14] & 98 & 30 & 2.3 & 39 & 11 \\
\hline Titche et al. [3] & 128 & 9.4 & 11 & 36 & 2.3 \\
\hline Parnell et al. [15] & 86 & 23 & 10.7 & 40.5 & 8.3 \\
\hline Terris et al. [16] & 84 & 34.5 & 10.7 & 29.9 & 8.4 \\
\hline Chen et al. [10] & 291 & 40.2 & 18 & 16 & 6 \\
\hline Our study & 50 & 34 & & & \\
\hline
\end{tabular}


Lung malignancies with secondaries are known to cause direct compression over the recurrent laryngeal nerve making it one of the common causes for left sided VC paralysis. Oesophageal and thyroid malignancies can also contribute to the same [14]. Chen et al showed lung cancer as a cause of VC paralysis in 34 cases [10]. Our study also reported Bronchogenic malignancy as the one of the common sites of tumor origin leading to vocal cord palsy. Even with the advent of high-quality imaging techniques, endoscopies and other investigations, idiopathic category is found to be the second most common cause of vocal cord palsy in our study [16\%]. Idiopathic, by definition, implies no exact cause identified. 10-27\% of reported cases in the English literature have an idiopathic origin, whereas the Japanese literature reports a rate of $25.9-41.3 \%$ [1]. Among the bilateral vocal cord paralysis patients, iatrogenic surgical cause due to cardiothoracic surgeries, spine surgeries and thyroidectomy were found to be maximum in our study. Neurological causes like cerebrovascular accidents, Parkinson's disease, autoimmune diseases like rheumatoid arthritis affecting the cricoarytenoid joint was reported to be another common etiology. Similar etiology has been discussed in various other studies too. Cerebrovascular accidents [stroke] due to uncontrolled hypertension can affect nucleus ambiguous and nucleus solitarius, the central connections of vocal cords, which can lead to vocal cord palsy. Diabetic patients are more prone to vocal cord palsy as they develop peripheral neuropathy $[9,15]$. Other rare causes like thymic enlargement, Ortner's syndrome, Zenker's diverticulum, diffuse idiopathic skeletal hyperostosis have also been reported as cause of vocal cord paralysis in few studies [16-22].

\section{Conclusion}

Vocal cord paralysis has a wide range of factors contributing to its occurrence with surgical cause being a common one. The advanced techniques and improved surgical skills can lead to reduction in surgeries being the cause of vocal cord paralysis. The incidence of unilateral vocal cord paralysis is more than bilateral vocal cord paralysis. A comprehensive assessment of patient should be performed to determine the cause which facilitates a direct treatment plan.

\section{References}

1. Myssiorek D (2004) Recurrent laryngeal nerve paralysis: anatomy and etiology. Otolaryngol Clin N Am 37: 25-44.

2. Furukawa M, Furukawa MK, Ooishi K (1994) Statistical analysis of malignant tumors detected as the cause of vocal cord paralysis. ORL J Otorhinolaryngol 56: 161-165.
3. Tucker HM (1980) Vocal cord paralysis etiology and management. Laryngoscope 90: 585-590.

4. Titche LL (1976) Causes of recurrent laryngeal nerve paralysis. Arch Otolaryngol 102: 259-261.

5. Bando H, Nishio T, Bamba H, Uno T, Hisa Y (2006) Vocal cord paralysis as a sign of chest diseases: a 15-year retrospective study. World J Surg 30: 293-298.

6. Michael S, Benninger, John B Gillen, Jerald S Altman (1998) Changing Etiology of Vocal Fold Immobility. Laryngoscope 108(9): 1346-1350.

7. Feehery JM, Pribitkin EA, Heffelfinger RN, Lacombe VG, Lee D, et al. (2003) The evolving etiology of bilateral vocal fold immobility. J Voice17(1): 76-81.

8. Gupta J, Varshney S, Bist SS, Bhagat S (2013) Clinico-etiological study of vocal cord paralysis. Indian J Otolaryngol Head Neck Surg 65(1): 16-19.

9. HT Anil, N Lasya Raj, Nikitha Pillai (2019) A Study on Etiopathogenesis of Vocal Cord Paresis and Palsy in a Tertiary Centre. Indian J Otolaryngol Head Neck Surg 71(3): 383-389.

10. Chen HC, Jen YM, Wang CH, Lee JC, Lin YS (2007) Aetiology of vocal cord paralysis. ORL J Otorhinolaryngol Relat Spec 69: 167-171.

11. Parnell FW, Barndenburg JH (1970) Vocal cord paralysis: a review of 100 cases. Laryngoscope 80(7): 1036-1045.

12. Hsin CC, Yee MJ, Chin HW, Jin CL, Yaoh SL (2007) Etiology of Vocal Cord Paralysis. ORL 69(3): 167-171.

13. Yumoto E, Minoda R, Hyodo M, Yamagata T (2002) Causes of recurrent laryngeal nerve paralysis. Auris Nasus Larynx 29: 41-45.

14. Toutounchi SJS, Eydi M, Golzari SEJ, Ghaffari MR, Parvizian N (2014) Vocal cord paralysis and its etiologies: a prospective study. J Cardiovasc Thorac Res 6(1): 47-50.

15. Misron K, Balasubramaniam A, Mohamad I, Hassan NFHN (2014) Bilateral vocal cord palsy post thyroidectomy: lessons learnt. BMJ Case Rep 2014: bcr2013201033.

16. Nuutinen J, Karia J (1981) Bilateral vocal cord paralysis following general anaesthesia. Laryngoscope 91(1): 83-86

17. Viswanath B, Pooja R (2018) A case of left vocal cord palsy due to thymic enlargement. American Journal of otolaryngology and head and neck surgery 1(6): 1028

18. Laura H, Rosenthal S, Benninger MS, Deeb RH (2007) Vocal fold immobility: a longitudinal analysis of aetiology over 20 years. Laryngoscope 117: 1864-1870.

19. Leder SB, Ross DA (2005) Incidence of vocal fold immobility in patients with dysphagia. Dysphagia 20(2): 168-169.

20. Ramadan HH, Wax MK, Avery S (1998) Outcome and changing cause of unilateral vocal cord paralysis. Otolaryngol Head Neck Surg 118: 199202.

21. Terris DJ, Arnstein DP, Nguyen HH (1992) Contemporary evaluation of unilateral vocal cord paralysis. Otolaryngol Head Neck Surg 107: 84-90.

22. Shruti Dhingra (2020) Rare Causes of Unilateral Vocal Fold Paralysis: Report of 3 Cases with Review of Literature. Indian J Otolaryngology Head Neck Surg. 
This work is licensed under Creative Commons Attribution 4.0 License

To Submit Your Article Click Here: $\quad$ Submit Article

DOI: $10.32474 /$ SJ0.2020.05.000219

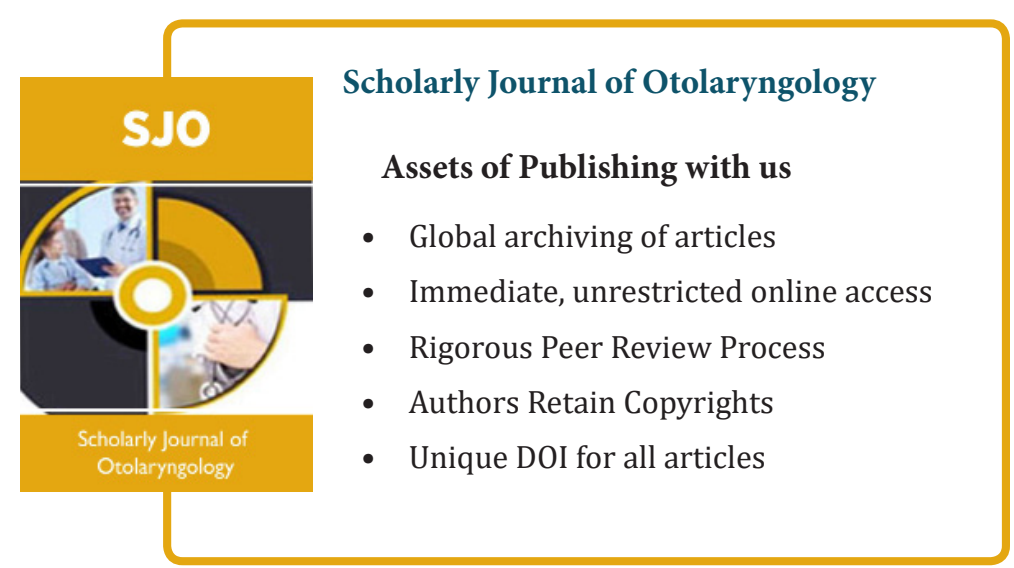

